\title{
Primary hyperparathyroidism versus familial hypocalciuric hypercalcemia: a challenging diagnostic evaluation in an adolescent female
}

\author{
Mustafa Tosur, MD', \\ Monica E. Lopez, MD', \\ David L. Paul, MD ${ }^{1}$ \\ ${ }^{1}$ Section of Diabetes and Endocri- \\ nology, Department of Pediatrics, Texas \\ Children's Hospital, Baylor College of \\ Medicine, Houston, TX, USA \\ ${ }^{2}$ Department of Surgery and Pediatrics, \\ Texas Children's Hospital, Baylor College \\ of Medicine, Houston, TX, USA
}

Primary hyperparathyroidism (PHPT) and familial hypocalciuric hypercalcemia (FHH) have significantly different treatment approaches, so physicians must be careful to differentiate these 2 diseases. Herein, we report a 14-year-old female who presented with symptomatic hypercalcemia $(12 \mathrm{mg} / \mathrm{dL}$; reference range, 9.2-10.7 $\mathrm{mg} / \mathrm{dL})$, elevated intact parathyroid hormone (iPTH) $(236 \mathrm{pg} / \mathrm{mL}$; reference range, 9-69 pg/mL), and vitamin D deficiency $(6 \mathrm{ng} / \mathrm{mL}$; reference range, $\geq 20 \mathrm{ng} / \mathrm{mL}$ ). On numerous occasions, her 24-hour urine calcium was more than $4 \mathrm{mg} / \mathrm{kg} / \mathrm{day}$, consistent with PHPT, but her fractional excretion of calcium on 24-hour urine collection was consistently below $1 \%$, in line with $\mathrm{FHH} .{ }^{99 \mathrm{~m}} \mathrm{Tc}-\mathrm{Sestamibi}$ scan failed to detect any abnormalities. However, a 4-dimensional computed tomography scan of the neck revealed a right superior parathyroid adenoma which was excised with a focused parathyroidectomy. Although the patient's calcium and iPTH levels normalized, her nonspecific symptoms persisted. This case illustrates both the challenges of differentiating PHPT from FHH and the limitations of a first-line imaging tool in identifying a parathyroid adenoma.

Keywords: Hypercalcemia, Primary hyperparathyroidism, Familial hypocalciuric hypocalcemia, Parathyroid adenoma, Parathyroid hormone

\section{Introduction}

Primary hyperparathyroidism (PHPT) is a rare disease in children with an estimated incidence of $2-5$ cases per 100,000 persons. ${ }^{1)}$ Whereas most adult patients with PHPT are asymptomatic, children with PHPT are usually symptomatic. ${ }^{2)}$ In contrast, familial hypocalciuric hypercalcemia $(\mathrm{FHH})$ is a relatively benign disease characterized by asymptomatic hypercalcemia. Nonetheless, the clinical presentations and biochemical findings of the 2 diseases may overlap significantly. Thus, distinguishing between these 2 entities is a crucial step in the evaluation of hypercalcemia. ${ }^{3)}$

Herein, we report a 14-year-old female who initially presented with hypercalcemia and an elevated intact parathyroid hormone (iPTH) level along with a description of the accompanying diagnostic challenges of differentiating PHPT from FHH.

\section{Case report}

A 14-year-old female presented to the emergency department with chest pain, eye pain, and headache. She was hypercalcemic $(12.1 \mathrm{mg} / \mathrm{dL}$; reference range, $9.2-10.7 \mathrm{mg} / \mathrm{dL})$ and hypertensive (systolic $131 \mathrm{mmHg}$; diastolic, $84 \mathrm{mmHg}$ ). A nephrology evaluation revealed elevated intact PTH $(236 \mathrm{pg} / \mathrm{mL}$; reference range, $9-69 \mathrm{pg} / \mathrm{mL})$, hypercalcemia $(13.2 \mathrm{mg} / \mathrm{dL}$ reference range, $9.2-10.7 \mathrm{mg} / \mathrm{dL})$ and vitamin D deficiency $(6 \mathrm{ng} / \mathrm{mL}$; reference range, $\geq 20$ 
$\mathrm{ng} / \mathrm{mL}$ ) with a normal basic metabolic panel, magnesium, and phosphorus. Ambulatory blood pressure monitoring over a 24hour period revealed hypertension, and she was started on a calcium channel blocker.

During the endocrinology evaluation, she reported the following symptoms: a 6-month history of fatigue; headache; abdominal pain; back pain; constipation; increased appetite; polydipsia $\left(2.3 \mathrm{~L} / \mathrm{m}^{2} /\right.$ day $)$; bone pain in her bilateral arms, hands, legs, and feet; muscle weakness; palpitations; and anxiety. She denied polyuria (despite excessive drinking), history of a kidney stone, or depression. The past medical and surgical histories were unremarkable. The patient's family history was significant for kidney stones in the maternal aunt, hypertension in the mother and maternal uncle, and diabetes in the maternal uncle. There was no known family history of calcium problems.

On physical examination, vital signs and anthropometric measurements were as follows: pulse rate, 76 beats $/ \mathrm{min}$; blood pressure, 138/91 mmHg (>99th percentile for both systolic and diastolic); weight, $60.3 \mathrm{~kg}$ (84th percentile); height, 161.6 $\mathrm{cm}$ (42nd percentile); and body mass index, $22.7 \mathrm{~kg} / \mathrm{m}^{2}$ (80th percentile). The physical examination revealed direct tenderness in the epigastric area.

The X-ray examinations of her left leg, left foot, and bilateral wrists did not show any fractures, periarticular erosions, or other bone lesions. Further biochemical tests confirmed elevated iPTH (188 pg/mL; reference range, 9-69 pg/mL) and hypercalcemia $(12.6 \mathrm{mg} / \mathrm{dL}$; reference range, $9.2-10.7 \mathrm{mg}$ / $\mathrm{dL}$ ). Spot urine $\mathrm{Ca} / \mathrm{Cre}$ ratios were $0.22,0.12$, and 0.11 , and the 24 -hour urine calcium values were 365,441 , and $278 \mathrm{mg}$ / day on 3 separate occasions. The 24-hour urine fractional excretions of calcium (FeCa) were $0.98 \%$ and $0.9 \%$ on 2 separate tests. Ultrasound of the kidneys revealed both kidneys were of normal size and appearance. Ultrasound of the neck and ${ }^{99 \mathrm{~m}} \mathrm{Tc}$-Sestamibi scan did not show a parathyroid adenoma. Multiple endocrine neoplasia 1 sequencing (Baylor Genetic Laboratories, Houston, TX, USA) and FHH next-generation sequencing panel with FHH-related genes (i.e., CASR, APP2S1, and GNA 11; Prevention Genetics, Marshfield, WI, USA) were negative. However, these results were not available at the time the treatment decision was made.

The pediatric surgical team recommended surgical intervention, and a preoperative 4-dimensional (4D) computed tomography (CT) scan of the neck revealed a right superior parathyroid adenoma (Fig. 1). Although the patient's symptoms and PTH levels suggested PHPT, the diagnosis was not confirmed until the identification of the parathyroid adenoma on the 4D CT scan of the neck. The right superior parathyroid gland was removed by focused parathyroidectomy, and a pathology examination revealed a parathyroid adenoma $(0.9 \mathrm{~g}$ in weight, $2.5 \mathrm{~cm}$ in greatest dimension). The calcium and iPTH levels normalized after surgery $(\mathrm{Ca}, 10.3 \mathrm{mg} / \mathrm{dL}$; reference range, 9.2-10.7 mg/dL and iPTH, $15 \mathrm{pg} / \mathrm{mL}$; reference range, 9-69 pg/ $\mathrm{mL}$ ) (Fig. 2).

However, the patient reported that her symptoms improved for only 1 week. She continued to complain of fatigue, headache, abdominal pain, bone pain, back pain, and polydipsia, along with the new complaint of polyuria. She also developed intermittently swollen feet and ankles. Follow-up calcium and iPTH levels were normal. Doppler ultrasound of her lower extremities showed bilateral idiopathic lower extremity edema without any deep venous thrombosis. Compression stockings

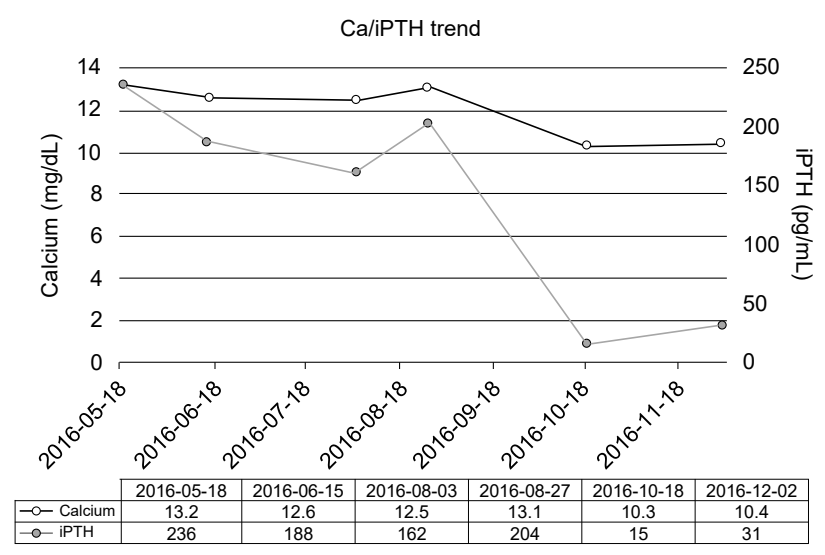

Fig. 2. Pre- and postoperative calcium and intact parathyroid hormone (iPTH) trends in parathyroid adenoma.

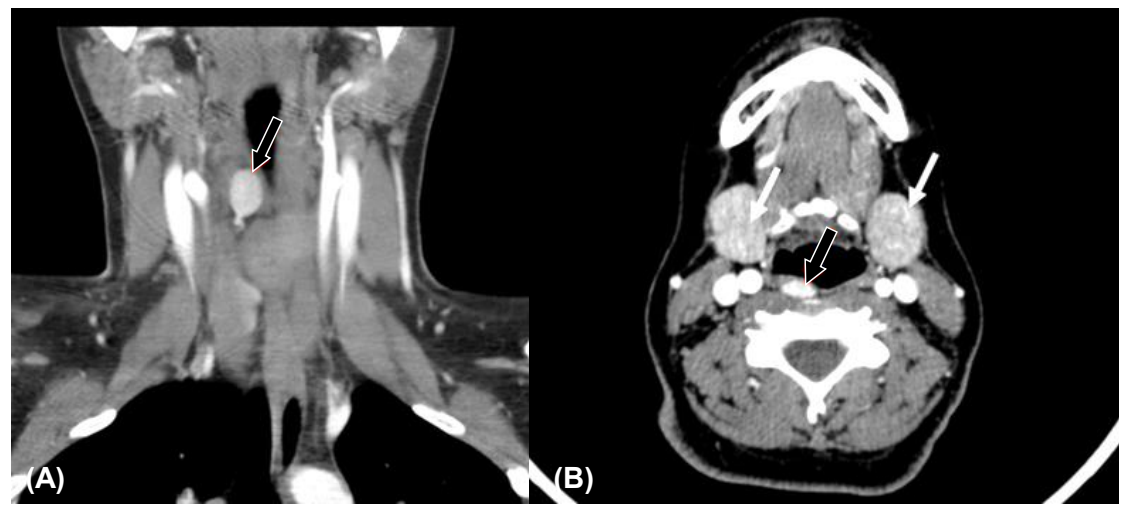

Fig. 1. Four-dimensional computed tomography scan of the neck revealed a right superior parathyroid adenoma (black arrow) and salivary glands (white arrow) in coronal (A) and transverse (B) images. 
were recommended, especially while the patient remained in an upright position.

One year after parathyroidectomy, the patient reported significant improvement of the lower extremity swelling, and she no longer requires compression stockings. In addition, the patient's postoperative medications include an antihypertensive and a combination of amitriptyline, topiramate, and sumatriptan for newly-diagnosed migraine headache.

\section{Discussion}

We report a challenging diagnostic evaluation of an adolescent female with hypercalcemia and an elevated iPTH level. Although the clinical presentations and biochemical findings may overlap in PHPT and FHH, clinicians must distinguish between the 2 entities because of the drastic differences in treatment. In the present case, the initial biochemical findings and imaging studies were inconclusive. Moreover, the patient's vitamin D deficiency likely complicated the clinical picture by affecting her excretion of urinary calcium. The definitive diagnosis of PHPT could only be made after a parathyroid adenoma was identified on a 4D CT scan of the neck.

Numerous factors have been proposed to help differentiate PHPT from FHH: symptomatology (symptomatic in PHPT), bone density (decreased in PHPT), family history of hypercalcemia (positive in $\mathrm{FHH}$ ), previous normocalcemia (present in PHPT), and FeCa values $(<1 \%$ in $\mathrm{FHH}$ and $>2 \%$ in PHPT) ${ }^{3,4)}$ Nonetheless, there is significant overlap in these differentiating factors. A prospective observational study assessed the diagnostic performance of FeCa in patients with genetically-confirmed FHH $(n=54)$ and patients with histologically-confirmed PHPT $(\mathrm{n}=97)$. Median FeCa ratios in 24 -hour urine samples were $0.6 \%$ (range, $0.2 \%-2.6 \%$ ) and $2.2 \%$ (range, $0.4 \%-4.8 \%$ ) in patients with FHH and PHPT, respectively. A cutoff point of $\mathrm{FeCa}<1 \%$ for $\mathrm{FHH}$ was found to sample $65 \%$ of patients with $\mathrm{FHH}$ and $4 \%$ of patients with PHPT. Approximately one-third of patients in both groups had FeCa values ranging between $1 \%$ and $2 \%$. These findings prompted the authors to suggest a 2-step diagnostic procedure: first, screen for FeCa cutoff of $<2 \%$ (which would sample $98 \%$ of patients with $\mathrm{FHH}$ and $35 \%$ of patients with PHPT); and second, perform FHH mutation analysis in those patients with FeCa values of $<2 \%$. ${ }^{5}$ Accordingly, the 24-hour urine FeCa value was $<1 \%$ in our patient. Furthermore, several factors, such as vitamin D deficiency and low intake of dietary calcium, may affect urinary calcium levels and should be kept in consideration. Interestingly, vitamin D deficiency is a common occurrence in patients with PHPT due to the negative feedback of $1,25-\mathrm{OH}$ vitamin $\mathrm{D}$ to dermal D3 production and increased 24-hydroxylation of 25-OH vitamin D. ${ }^{6}$ Vitamin D deficiency in our patient likely contributed to the low FeCa value and rendered differentiating between PHPT and FHH more difficult with the initial biochemical tests.

${ }^{99 \mathrm{~m}} \mathrm{Tc}$-Sestamibi scan is the preoperative imaging modality most commonly used for PHPT. In a systematic review of 215 studies $(n=20,225)$, the sensitivities of ${ }^{99 m}$ Tc-Sestamibi scan and high-resolution ultrasound were reported as follows: $88.44 \%$ vs. $78.55 \%$ for solitary adenomas, $44.46 \%$ vs. $34.86 \%$ for multiple-gland hyperplasia, $29.95 \%$ vs. $16.2 \%$ for double adenomas, and $33 \%$ vs. $100 \%$ for carcinomas, respectively. ${ }^{7)}$ Another review reported the sensitivities of the sestamibi scan, ultrasound, 4D CT, and magnetic resonance imaging as 78.9\%, $76.1 \%, 89.4 \%$, and $43 \%$ to $71 \%$, respectively (8). In patients requiring reoperation for hyperparathyroidism, a retrospective study $(n=45)$ at MD Anderson Cancer Center showed that the sensitivity for localization was $88 \%$ for $4 \mathrm{D}$ CT, whereas it was $44 \%$ for sestamibi scan and $21 \%$ for ultrasound. ${ }^{8)}$ However, the risk-benefit ratio should be carefully considered when obtaining a 4D CT scan given the significant radiation exposure to the thyroid gland. ${ }^{9}$ Although a sestamibi scan failed to identify the solitary right parathyroid adenoma in our patient, a 4D scan exposed the lesion.

Interestingly, the patient's nonspecific symptoms persisted even after normalization of her serum calcium level. The persistence of her symptoms could be partly explained by her concomitant health problems, including hypertension, migraine, and idiopathic edema in her lower extremities. Although her medication for hypertension was discontinued shortly after the removal of the parathyroid adenoma, it was restarted a few months later due to significantly high blood pressure readings on ambulatory blood pressure monitoring. The exact cause of her persistent hypertension was unknown, but it was likely essential hypertension. She continued to require several medications to manage her migraine, which may have contributed to her persistent fatigue. The lack of polyuria despite the presence of polydipsia at the initial presentation may be due to reporting bias of the patient. Even at her initial presentation, we suspect she had polyuria based on the reported fluid intake. We speculate that the persistence of polyuria/polydipsia after normalization of the calcium levels could be due to psychogenic polydipsia.

In conclusion, it is crucial to differentiate PHPT from $\mathrm{FHH}$ because of the dramatic differences in treatment. Inconclusive imaging studies do not rule out PHPT. In cases with a strong clinical suspicion, appropriate further imaging and/or surgical intervention should be performed.

\section{Ethical statement}

An informed consent was obtained from patient's parent.

\section{Conflict of interest}

No potential conflict of interest relevant to this article was reported. 


\section{Acknowledgments}

The authors thank Dr. B. Lee Ligon, Centers for Research, Innovation and Scholarship, Department of Pediatrics, Baylor College of Medicine, for editorial assistance.

\section{References}

1. Roizen J, Levine MA. Primary hyperparathyroidism in children and adolescents. J Chin Med Assoc 2012;75:42534.

2. Tosur M, Patel KN, Swischuk LE, Lee PD. Incidental hypercalcemia caused by primary hyperparathyroidism with rapid progression to renal complications in a child. Clin Pediatr (Phila) 2018;57:117-20.

3. Shinall MC Jr, Dahir KM, Broome JT. Differentiating familial hypocalciuric hypercalcemia from primary hyperparathyroidism. Endocr Pract 2013;19:697-702.

4. Bilezikian JP, Potts JT Jr, Fuleihan Gel-H, Kleerekoper M, Neer R, Peacock M, et al. Summary statement from a workshop on asymptomatic primary hyperparathyroidism: a perspective for the 21st century. J Clin Endocrinol Metab 2002;87:5353-61.
5. Christensen SE, Nissen PH, Vestergaard P, Heickendorff L, Brixen K, Mosekilde L. Discriminative power of three indices of renal calcium excretion for the distinction between familial hypocalciuric hypercalcaemia and primary hyperparathyroidism: a follow-up study on methods. Clin Endocrinol (Oxf) 2008;69:713-20.

6. Moosgaard B, Vestergaard P, Heickendorff L, Melsen F, Christiansen P, Mosekilde L. Vitamin D status, seasonal variations, parathyroid adenoma weight and bone mineral density in primary hyperparathyroidism. Clin Endocrinol (Oxf) 2005;63:506-13.

7. Ruda JM, Hollenbeak CS, Stack BC Jr. A systematic review of the diagnosis and treatment of primary hyperparathyroidism from 1995 to 2003. Otolaryngol Head Neck Surg 2005; 132:359-72.

8. Mortenson MM, Evans DB, Lee JE, Hunter GJ, Shellingerhout D, Vu T, et al. Parathyroid exploration in the reoperative neck: improved preoperative localization with 4D-computed tomography. J Am Coll Surg 2008;206:88895.

9. Kunstman JW, Kirsch JD, Mahajan A, Udelsman R. Clinical review: parathyroid localization and implications for clinical management. J Clin Endocrinol Metab 2013;98:902-12. 